\title{
RAF1 amplification: an exemplar of MAPK pathway activation in urothelial carcinoma
}

\author{
Sean Clark-Garvey ${ }^{1}$ and William Y. Kim ${ }^{1,2,3,4}$ \\ 'Division of Oncology, Department of Medicine; ${ }^{2}$ Lineberger Comprehensive Cancer Center; ${ }^{3}$ Department of Pharmacology; and ${ }^{4}$ Department of Cenetics, University of North Carolina at Chapel Hill, \\ Chapel Hill, North Carolina, USA.
}

\begin{abstract}
Despite recent therapeutic gains in the treatment of advanced bladder cancer, the overall survival in patients with metastatic disease remains poor and further therapeutic discovery is needed. Advanced bladder cancer is a molecularly heterogeneous disease, and the identification of driver genetic alterations has led to effective targeted therapeutic agents, such as fibroblast growth factor receptor (FCFR) inhibitors. In this issue of the $J C I$, Bekele et al. identify a subtype of muscle-invasive bladder cancer (MIBC) that harbors RAF1 amplification. The authors showed that RAF1 inhibition, with pan-RAF inhibitors, and the combination of RAF1 inhibition with MEK inhibition were efficacious in preclinical models harboring RAF1 amplifications as well as in tumors with HRAS and NRAS mutations. This study highlights RAF1 amplification as a driver event in bladder cancer and establishes the central role of the MAPK pathway in bladder tumorigenesis.
\end{abstract}

\section{Bladder cancer and the MAPK pathway}

In $2021,83,730$ cases of bladder cancer are expected, making it the sixth most common cause of cancer in the United States, which will result in an estimated 17,200 deaths (1). While there have been important advances in the treatment of advanced bladder cancer over the past decade, the mortality associated with metastatic disease remains particularly grim. Cisplatin-based regimens have long been the mainstay of firstline therapy in this setting. More recently, the development of other systemic therapies, including immunotherapy (specifically PD-1 axis inhibitors), antibody drug conjugates, and fibroblast growth factor receptortargeted (FGFR-targeted) therapy have also proven effective (2). Nonetheless, overall survival with these regimens remains on the order of 10 to 14 months, highlighting the need for other effective therapeutic agents/combinations.

The MAPK pathway (in this case, RAF/ MEK/ERK; Figure 1A) is a signaling cascade that links extracellular signals to processes, such as cell differentiation, proliferation, and growth. Dysregulation of the MAPK pathway through various means has been identified across a multitude of cancers, with the RAS and RAF proteins in particular having a high frequency of activating mutations (3). The RAS family of proteins, consisting of KRAS, NRAS, and HRAS, are guanine triphosphate-hydrolyzing (GTP-hydrolyzing) enzymes that cycle between an active GTP-bound and an inactive guanine diphosphate-bound (GDP-bound) state. RAS mutations impair GTP hydrolysis, promoting a GTP-bound

Delated Article: https://doi.org/10.1172/JCl147849

Conflict of interest: WYK receives research support from Acerta, GeneCentric, and Merck and has ownership in AbbVie, Abbott, Amgen, Arvinas, BeiGene, Bluebird Bio, Bristol Myers Squibb, Myovant, Natera, Oramed, Zentalis, and ACT (Advanced Chemotherapy Technologies).

Copyright: () 2021, American Society for Clinical Investigation.

Reference information: / Clin Invest. 2021;131(22):e154095. https://doi.org/10.1172/JCl154095.

state and downstream activation of the RAF, MEK, and ERK kinases (3). The RAF family of kinases consists of serine/threonine kinases with three known isoforms in humans (ARAF, BRAF, and RAF1, also known as CRAF). BRAF is the isoform most commonly mutated in cancer, most notably in the $B R A F^{V 600 E}$ mutation, which occurs in a number of different cancers, including melanoma and colorectal cancer, nonsmall cell lung cancer (NSCLC), and thyroid cancer (4). BRAF inhibitors are particularly effective in $B R A F^{V 600 E}$ mutated malignancies, especially when administered in combination with mitogen-activated protein kinase (MEK) inhibitors, and there are now multiple FDA-approved BRAF inhibitors and BRAF/MEK inhibitor combinations (4).

\section{RAF1 is amplified in bladder cancers}

Unlike $B R A F, R A F 1$ is rarely mutated in cancer, perhaps secondary to its low basal kinase activity (relative to BRAF) as well as the need for posttranslational modification for activation $(4,5)$. In this issue of the JCI, Bekele et al. note that $12 \%$ of muscle-invasive bladder cancers (MIBCs) harbor focal amplification of RAF1. Notably, an analysis across TCGA cancer types shows that MIBCs have the highest rate of RAF1 amplification of all tumors, with the next most frequent tumor type being sarcoma (approximately 3\%; ref. 6). The authors made a number of interesting genomic associations, including that $R A F 1$ amplification was enriched in the luminal unstable (LumU) consensus molecular subtype (35\% of RAF1-amplified tumors are classified as LumU; refs. 6, 7) and that RAF1-amplified MIBCs had a significantly higher total mutation count than non-RAF1-amplified tumors. Additionally, RAF1-amplified tumors cooccurred with PPARG amplification (also on chromosome $3 p)$ and $E 2 F 3$ amplification, and there was a trend toward cooccurrence with TP53 
A

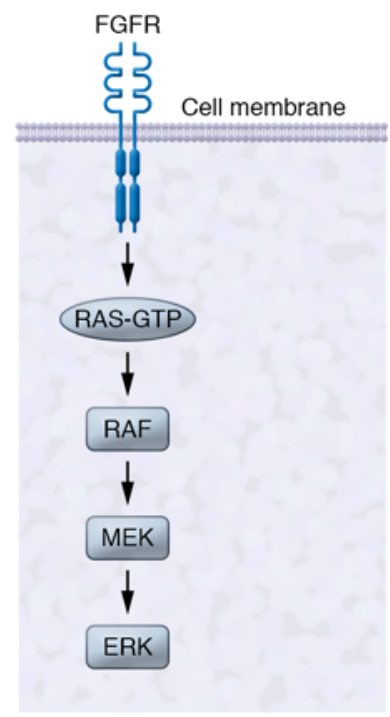

B

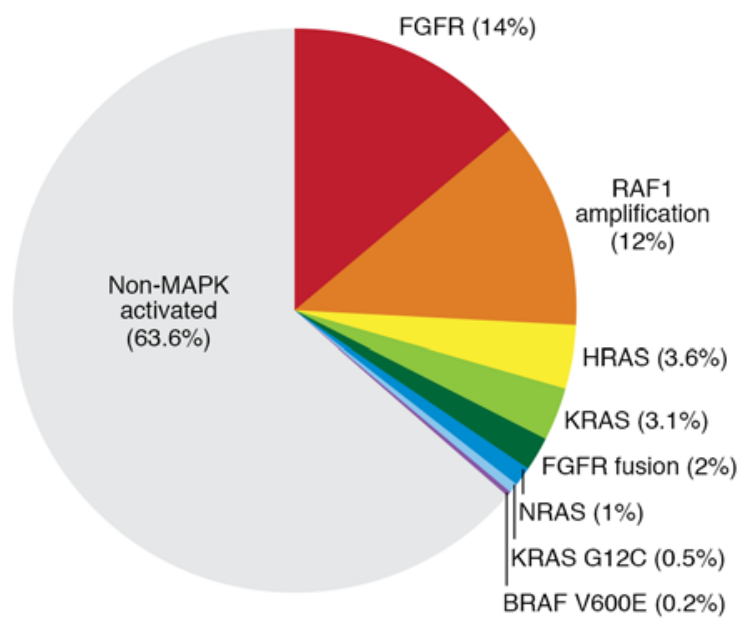

C

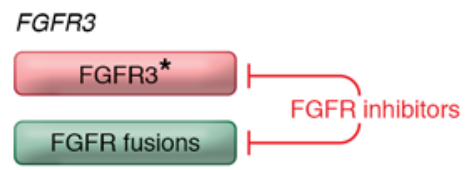

RAS

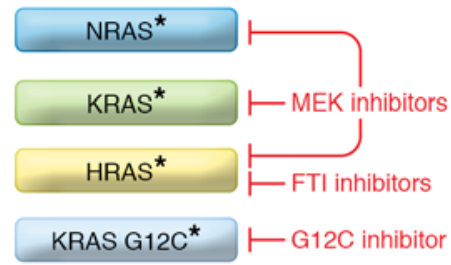

RAF

BRAF V600E* $\vdash$ BRAF inhibitors

RAF1 amplification $\longmapsto$ Pan-RAF inhibitors

Figure 1. MAPK activation and targeting in bladder cancer. (A) The RAF/MEK/ERK signaling cascade links extracellular signals to cell differentiation, proliferation, and growth. (B) The Cancer Genome Atlas (TCCA) indicates that MAPK alterations make up $36.4 \%$ of high-grade MIBCs. (C) Specific inhibitors may differentially target putative genomic alterations that activate the MAPK pathway to treat bladder tumors. Asterisks indicate mutations.

alterations. Conversely, the RAF1-amplified tumors and FGFR3 mutations were mutually exclusive (6).

To investigate the functional relevance of RAF1 amplification, Bekele et al. performed a series of elegant in vitro experiments and analyses of publicly available essentiality data sets. For example, siRNA of $R A F 1$ in bladder cancer cell lines with known $R A F 1$ amplification decreased MAPK signaling as well as in vitro proliferation. Query of the publicly available DepMap data showed that the RAF1-amplified 5637 cell line was highly sensitive to pharmacological RAF inhibition with the pan-RAF inhibitor RAF265 as well as the BRAFV600E inhibitor PLX4720. Importantly, bladder cancer cell lines without $R A F 1$ amplification showed no dependence upon RAF1. Given the known success of concurrent targeting of BRAF and MEK in MAPK-activated cancers, the authors showed that RAF1-amplified cell lines were highly sensitive to dual targeting of RAF1 and MEK by RAF265 and trametinib. Finally, the researchers validated their findings in two independent in vivo models: UMUC9 xenografts as well as a patientderived xenograft, with known RAF1 amplification. The combination of RAF265 and trametinib potently reduced tumor size and increased immunohistochemical staining for cleaved PARP consistent with an increase in cell death (6).

\section{RAS mutations activate the MAPK pathway}

While RAS mutations are uncommon in MIBCs, activating mutations in KRAS, HRAS, and NRAS do occur in a small percentage of patients (Figure 1B). Given the established importance of RAF and especially RAF1 in signaling downstream of RAS isoforms, Bekele et al. hypothesized that targeting RAF with or without MEK inhibition would effectively reduce NRAS and HRAS mutant tumors. They found that T24 cells (HRAS G12V mutant) and Ku-19-19 cells (NRAS Q61R mutant) were highly sensitive to RAF265 alone or in combination with trametinib (6). These studies, albeit in a single cell line, identify a further therapeutic role for dual RAF1 and MEK inhibition in RAS mutant bladder cancer. Nonetheless, these impressive preclinical results should be viewed cautiously, since the use of RAF and MEK inhibitors in RAS mutant cancers has shown variable clincial results. For example, the MEK inhibitor binimetinib demonstrated a progression-free survival benefit over dacarbazine in NRAS mutant melanoma (8), while trametinib did not show superiority over docetaxel in KRAS mutant NSCLC (9).

\section{The MAPK pathway is}

\section{targetable in bladder cancer}

Bekele and colleagues describe RAF1amplified bladder cancers as another subset of MIBC. Moreover, their studies also demonstrate that bladder tumors with NRAS and HRAS mutations are sensitive to dual RAF/MEK inhibition. Their work elegantly highlights a key role of the MAPK pathway, which is activated in a considerable proportion of bladder cancers (i.e., $R A F 1$ amplification, BRAF ${ }^{V 600 E}$ mutation, and RAS [KRAS, HRAS, NRAS] mutations; Figure $1 \mathrm{~B}$ and ref. 6). These findings are perhaps unsurprising, given the early recognition that HRAS mutations occur in bladder cancer cell lines (10-12), reports of low-frequency activating $B R A F^{V 600 E}$ mutations in high-grade bladder cancers (13), and the MEK/ERK pathway as the predominant signaling pathway downstream of FGFR3 in urothelial cells (14). Furthermore, the fact that $R A F 1$ amplification is mutually exclusive with FGFR3 alterations and that RAF1-amplified tumors are enriched in the LumU consensus molecular subtype also underscores the importance of the RAF/MEK/ERK pathway in both bladder tumorigenesis and the signaling underlying development of the luminal molecular subtype $(15,16)$. Indeed, Bekele et al.'s work nicely emphasizes how we might invoke a more granular molecular stratification of driver events in MIBC (Figure 1). Despite the fact that these alterations all converge to activate RAF/MEK/ERK signaling, we propose that they each may be targeted differently given the development of therapies 
such as farnesyl transferase inhibitors and RAS G12C inhibitors (Figure 1C).

\section{Does RAF1 amplification promote immune-checkpoint blockade resistance?}

Notably, the highest frequency of RAF1 amplification was seen in the consensus LumU subtype, which has considerable overlap with the genomically unstable (GU) subtype previously defined by the Lund classification (17). The Lund GU subtype responds best to the anti-PD-L1 antibody atezolizumab, with approximately 50\% of patients with Lund GU tumors demonstrating a partial or complete response to atezolizumab (18). Given the evolving evidence that RAS/RAF/MEK pathway activation promotes immune evasion and subsequent resistance to $\mathrm{PD}-1 / \mathrm{PD}-\mathrm{L} 1$ inhibitors $(19,20)$, one intriguing possibility is that the subset of Lund GU tumors that are nonresponsive to immune-checkpoint blockade (ICB) are those with RAS/RAF/MEK pathway activation. If true, ICB response could be augmented by combined MEK inhibition and PD-1 axis blockade.

\section{Conclusions}

In conclusion, the work by Bekele et al. underscores the importance of continued exploration into driver events underlying MIBC. The identification of this novel RAF1-amplified subtype that is highly dependent upon downstream MAPK signaling underscores the importance of this pathway in bladder tumorigenesis and perhaps development of the luminal molecular subtype. Further investigation will hopefully resolve questions such as whether RAF1 amplification itself drives luminal differentiation in urothelial cells or whether this association is due to coamplification of PPARG, to what extent MAPK activation by these driver events (Figure 1B) fosters an immune-excluded phenotype that can be reversed by MEK inhibition, and the ultimate clinical efficacy of dual RAF/MEK inhibition in RAF1-amplified bladder cancers.

\section{Acknowledgments}

WYK is supported by the University Cancer Research Fund (UCRF) and by the NIH under award number R01-CA241810. SCG is supported by National Cancer Institute grant T32-CA244125.

Address correspondence to: William Y. Kim, Lineberger Comprehensive Cancer Center, University of North Carolina, CB\# 7295, Chapel Hill, North Carolina 275997295, USA. Phone: 919.966.4765; Email: wykim@med.unc.edu.

1. Siegel RL, et al. Cancer Statistics, 2021. Ca Cancer JClin. 2021;71(1):7-33.

2. Patel VG, et al. Treatment of muscle-invasive and advanced bladder cancer in 2020. CA Cancer JClin 2020;70(5):404-423.

3. Dhillon AS, et al. MAP kinase signalling pathways in cancer. Oncogene. 2007;26(22):3279-3290.

4. Holderfield M, et al. Targeting RAF kinases for cancer therapy: BRAF-mutated melanoma and beyond. Nat Rev Cancer. 2014;14(7):455-467.

5. Emuss V, et al. Mutations of C-RAF are rare in human cancer because C-RAF has a low basal kinase activity compared with B-RAF. Cancer Res. 2005;65(21):9719-9726.

6. Bekele RT, et al. RAF1 amplification drives a subset of bladder tumors and confers sensitivity to MAPK-directed therapeutics. JClin Invest. 2021;131(22):e147849.

7. Kamoun A, et al. A consensus molecular classification of muscle-invasive bladder cancer. Eur Urol. 2019;77(4):420-433.

8. Dummer R, et al. Binimetinib versus dacarbazine in patients with advanced NRAS-mutant melanoma (NEMO): a multicentre, open-label, randomised, phase 3 trial. Lancet Oncol. 2017;18(4):435-445.

9. Blumenschein GR, et al. A randomized phase II study of the MEK1/MEK2 inhibitor trametinib (GSK1120212) compared with docetaxel in KRAS-mutant advanced non-small-cell lung cancer (NSCLC) ${ }^{\dagger}$. Ann Oncol. 2015;26(5):894-901.

10. Taparowsky E, et al. Activation of the T24 bladder carcinoma transforming gene is linked to a single amino acid change. Nature. 1982;300(5894):762-765.

11. Reddy EP, et al. A point mutation is responsible for the acquisition of transforming properties by the T24 human bladder carcinoma oncogene. Nature. 1982;300(5888):149-152

12. Tabin CJ, et al. Mechanism of activation of a human oncogene. Nature. 1982;300(5888):143-149.

13. Iyer G, et al. Prevalence and co-occurrence of actionable genomic alterations in high-grade bladder cancer. JClin Oncol. 2013;31(25):3133-3140.

14. di Martino E, et al. Mutant fibroblast growth factor receptor 3 induces intracellular signaling and cellular transformation in a cell type- and mutation-specific manner. Oncogene. 2009;28(48):4306-4316

15. Damrauer JS, et al. Intrinsic subtypes of highgrade bladder cancer reflect the hallmarks of breast cancer biology. Proc Natl Acad Sci U S A. 2014;111(8):3110-3115

16. Choi W, et al. Identification of distinct basal and luminal subtypes of muscle-invasive bladder cancer with different sensitivities to frontline chemotherapy. Cancer Cell. 2014;25(2):152-165.

17. Sjödahl G, et al. A molecular taxonomy for urothelial carcinoma. Clin Cancer Res. 2012;18(12):3377-3386.

18. Mariathasan S, et al. TGF $\beta$ attenuates tumour response to $\mathrm{PD}$-L1 blockade by contributing to exclusion of T cells. Nature. 2018;554(7693):544-548.

19. Loi S, et al. RAS/MAPK activation is associated with reduced tumor-infiltrating lymphocytes in triple-negative breast cancer: therapeutic cooperation between MEK and PD-1/PD-L1 immune checkpoint inhibitors. Clin Cancer Res. 2016;22(6):1499-1509.

20. Hugo W, et al. Genomic and transcriptomic features of response to anti-PD-1 therapy in metastatic melanoma. Cell. 2016;165(1):35-44 